\title{
The use of the Panda-Nerve Block pain app in single-shot peripheral nerve block patients: a feasibility study
}

\section{Utilisation de l'application Panda pour la douleur suite à un bloc nerveux chez les patients recevant un bloc nerveux périphérique à injection unique : une étude de faisabilité}

\author{
Alexander Dotto, MD (D) - Dustin Dunsmuir, MSc - Terri Sun, MD, FRCPC • \\ Lily Y. L. Chiu, MD • Ronald Ree, MD, FRCPC • J. Mark Ansermino, MBBCH, FRCPC • \\ Cynthia H. Yarnold, MD, FRCPC
}

Received: 3 October 2019/Revised: 4 March 2020/Accepted: 15 March 2020/Published online: 4 June 2020

(C) Canadian Anesthesiologists' Society 2020

\begin{abstract}
Purpose Peripheral nerve blocks (PNBs) provide excellent perioperative analgesia but can increase the risk of severe postoperative pain once the block wears off. Poor adherence to discharge instructions may increase this risk. Panda-Nerve Block (Panda) is an app that alerts the patient to assess their PNB, score their pain, and take scheduled pain medication. We assessed the usability and feasibility of Panda for assisting patients after receiving a PNB.

Methods Twenty-nine patients tested Panda in three rounds, for two to seven days, postoperatively to assess and manage their pain and PNB. Feedback was provided via phone interview and the Computer System Usability Questionnaire (CSUQ). Additionally, each user's usage log
\end{abstract}

\footnotetext{
A. Dotto, MD $(\bowtie)$

Department of Anesthesiology, Pharmacology \& Therapeutics,

University of British Columbia, Vancouver, BC, Canada

e-mail: alex.dotto@alumni.ubc.ca

School of Population and Public Health, University of British

Columbia, Vancouver, BC, Canada

D. Dunsmuir, MSc

Department of Anesthesiology, Pharmacology \& Therapeutics,

University of British Columbia, Vancouver, BC, Canada

BC Children's Hospital Research Institute, Vancouver, BC,

Canada

T. Sun, MD, FRCPC $\cdot$ R. Ree, MD, FRCPC .

C. H. Yarnold, MD, FRCPC

Department of Anesthesiology, Pharmacology \& Therapeutics,

University of British Columbia, Vancouver, BC, Canada
}

was analyzed for parameters such as alert response times. Feasibility was determined by alert responses that occurred before the next alert, with a goal of greater than 50\%. User adherence was measured as percentage compliance with alerts within one hour; usability and user satisfaction were determined from the CSUQ and interviews.

Results A median [interquartile range (IQR)] of 68 [34$937 \%$ responded before the next alert during the first $48 \mathrm{hr}$ of app use, and 83 [54-92]\% responded before the next alert with 87 [75-96]\% of these within one hour. There were no significant differences in usage between rounds. Ninety-three percent of patients reported Panda to be easy to use and helpful, and $79 \%$ of patients would use Panda again. Critical themes included changes to the layout and appearance, clarification of the language of the PNB

Department of Anesthesia, Providence Health Care, St. Paul's Hospital, Vancouver, BC, Canada

\section{Y. L. Chiu, MD}

Department of Anesthesiology, Pharmacology \& Therapeutics, University of British Columbia, Vancouver, BC, Canada

J. M. Ansermino, MBBCH, FRCPC

Department of Anesthesiology, Pharmacology \& Therapeutics, University of British Columbia, Vancouver, BC, Canada

BC Children's Hospital Research Institute, Vancouver, BC, Canada

Department of Pediatric Anesthesia, BC Children's Hospital, Vancouver, BC, Canada 
check, and requests for dynamic adjustments to the medication schedule based on user responses.

Conclusion Panda-Nerve Block is a feasible method for PNB patients to manage postoperative pain with a high response rate. Future work should include providing twoway communication for patients and clinicians and assessing its effect on pain outcomes.

Trial registration $w w w$.clinicaltrials.gov

(NCT03369

392); registered 5 December 2017.

\section{Résumé}

Objectif Les blocs nerveux périphériques (BNP) procurent une excellente analgésie périopératoire mais peuvent augmenter le risque de douleur postopératoire élevée une fois que le bloc disparait. Un mauvais respect des instructions de congé pourrait augmenter ce risque. L'application Panda (Panda-Nerve Block) avertit le patient afin qu'il évalue son BNP, quantifie sa douleur, et prenne ses médicaments analgésiques prescrits. Nous avons évalué la facilité d'utilisation et la faisabilité de l'application Panda pour aider les patients ayant reçu un $B N P$.

Méthode Vingt-neuf patients ont testé l'application Panda en trois itérations de deux à sept jours après leur opération afin d'évaluer et de prendre en charge leur douleur et le $B N P$. Les rétroactions étaient partagées par entretien téléphonique et via le Questionnaire sur la convivialité du système informatique (CSUQ - Computer System Usability Questionnaire). En outre, le journal d'utilisation de chaque utilisateur a été analysé pour en étudier certains paramètres tels que les temps de réponse aux alertes. La faisabilité était déterminée par les réponses aux alertes survenant avant la prochaine alerte, avec un objectif de plus de $50 \%$. L'observance des utilisateurs était mesurée en tant que pourcentage de conformité aux alertes dans l'heure suivante; la facilité d'utilisation et la satisfaction des utilisateurs étaient déterminées à partir du CSUQ et des entretiens.

Résultats En moyenne [écart interquartile (ÉIQ)], 68 [34-93] \% des patients ont répondu avant la prochaine alerte au cours des premières $48 h$ d'utilisation de l'application, et 83 [54-92] \% ont répondu avant la prochaine alerte, avec 87 [75-96] \% de ces patients dans l'heure qui suivait. Il n'y a pas eu de différence significative dans l'utilisation entre les itérations. Quatre-vingt-treize pour cent des patients ont rapporté qu'ils trouvaient l'application Panda conviviale et utile, et $79 \%$ l'utiliseraient à nouveau. Les critiques comprenaient des modifications de la disposition et de l'apparence de l'application, la clarification $d u$ langage lors des vérifications du BNP, et des demandes pour des ajustements dynamiques du traitement selon les réponses des utilisateurs.
Conclusion L'application Panda constitue une méthode possible de prise en charge de la douleur postopératoire pour les patients ayant reçu un BNP, avec un taux de réponse élevé. Les travaux futurs devraient inclure la fourniture d'une communication bidirectionnelle pour les patients et les cliniciens et l'évaluation de l'effet de l'utilisation de l'application sur des devenirs de douleur. Enregistrement de l'étude $w w w$.clinicaltrials.gov (NCT 03369392); enregistrée le 5 décembre 2017.

Keywords regional $\cdot$ pain $\cdot$ smartphone $\cdot$ app

Peripheral nerve blocks (PNBs) are an appealing technique in day surgery patients because of their efficiency, reduced cost, and excellent perioperative pain management. ${ }^{1}$ Nevertheless, a recent study showed PNB patients were more likely to experience severe pain and to return to hospital for pain control than those who received a general anesthetic, ${ }^{2}$ findings consistent with other published results. ${ }^{3,4}$ Although patients are instructed to begin oral analgesia before their PNB resolves, many struggle with medication management; this may be because of an incomplete understanding of instructions, dosing difficulties, or poor motivation to take medication while a residual blockade remains. ${ }^{2}$ Critically, our clinicians report that many patients have poor recall of when the PNB wore off, confounding follow-up assessment.

Smartphone applications (apps) show promise in increasing therapy adherence. ${ }^{5}$ Patient-facing apps have been described and implemented for reducing anxiety during induction of anesthesia for children, ${ }^{6}$ selfmanagement of asthma ${ }^{7}$ and diabetes, ${ }^{8}$ adherence to an enhanced recovery after surgery protocol, ${ }^{9}$ and burn recovery. ${ }^{10}$

Panda (pain assessment via a novel digital app), an app developed by the Digital Health Innovation Lab at British Columbia Children's Hospital, is usable and feasible for parents managing their child's postoperative pain. ${ }^{11,12}$ This app has undergone extensive testing with patients' parents, patients, and nurses. A version of the app, Panda-Nerve Block (hereafter "Panda"), was created for adult PNB patients and includes features for monitoring a PNB. The app includes both the visual analogue scale (VAS) and numeric rating score (NRS) pain scales ${ }^{13,14}$; accordance between the paper and smartphone versions of these scales has been previously shown. ${ }^{15} \mathrm{We}$ aimed to assess the usability and feasibility of Panda in adult day surgery patients who received a single-shot PNB. 


\section{Methods}

\section{Study design}

The primary objective of this study was to show the feasibility of Panda with patients at home; the app was considered feasible if at least $50 \%$ of alerts were responded to by users before the next alert occurred. Additionally, alert response time within one hour was used as a measure of adherence. Our secondary objective was to assess usability and identify issues that arose at home to make iterative improvements to the app between rounds of testing. Feasibility is an assessment of whether or not patients will use Panda in practice. In this study, feasibility was measured by adherence to alerts and patient-reported satisfaction with the app. Usability is a measure of how easy and intuitive Panda was to use and whether there were issues that led to user confusion or undesirable outcomes.

Three separate rounds of testing were conducted in December 2017, February 2018, and May 2018, lasting for approximately two weeks each. This protocol was approved by the University of British Columbia Providence Health Care Research Ethics Board (REB Number H17-01365). We aimed to recruit ten patients per round for a total of 30 patients. This sample size was selected as it has been used in similar studies. ${ }^{11,12,16}$

\section{Selection and description of participants}

Patients were recruited in the surgical daycare unit and surgical procedure room, an ambulatory surgery unit at St. Paul's Hospital, a tertiary academic site in Vancouver, Canada. The inclusion criteria were American Society of Anesthesiologists physical status I-III, age 19-75 years, undergoing ambulatory surgery under PNB, and anticipated post-surgical pain. Patients were required to use their own iOS or Android device, to be discharged on the same day as their surgery, and have at least two days' planned post-discharge analgesic medication. Patients were excluded if they were unable to follow study instructions and complete questionnaires in English, did not have an iOS or Android device, or if there was a significant cognitive impairment, hearing or visual impairment, neurologic injury, or psychomotor dysfunction that precluded app usage. Eligible patients were approached by study personnel after being identified on the operating room slate. Written informed consent was obtained from each participant.

\section{Panda app}

The original Panda app has been described previously. ${ }^{11}$ It allows parents to schedule alert reminders in concordance with their child's prescription (Fig. 1). Several new features were designed specifically for Panda.

Initial configuration of the app includes demographic information, surgical information, and, specific to Panda, block information (location, presence of a catheter, concentration and volume of local anesthetic injected, and any adjuvant medication). Compared with the pediatric app, the Panda user is the patient rather than a parent. Both the NRS and VAS are available to users for pain assessment.

The pain check function was modified from the original Panda app to include questions about the quality of the patient's PNB. At each alert, patients are asked if their operative site has motor weakness and if the sensation is numb, tingly, or has returned. Patients are then asked where their pain is and to rate their pain. Patients may perform a pain and block check in between scheduled alerts, as well as log any breakthrough medications. These questions were determined by expert consensus at our institution and were based on our routine follow-up questions.

A tutorial and demo mode were available for practice. The upload function uploaded all the patient's Panda usage data to a Research Electronic Data Capture (REDCap) database on BC Children's Hospital Research Institute servers. ${ }^{17}$

\section{Data collection}

Patients completed a pre-study questionnaire pertaining to past experiences with smartphones and apps (Appendix 1). Participants were assisted in downloading, installing, and setting up the app on their own device. To assure roughly equal numbers of patients testing the VAS and NRS, patients were assigned to either scale in a non-randomized, alternating fashion; however, users were still able to switch scales if they preferred. To be consistent with our institution's discharge directions given to PNB patients, all prescribed analgesic medications were scheduled as a regular medication for the first $24 \mathrm{hr}$ post-discharge regardless of how the prescription had been written; medications were subsequently scheduled as either regular or PRN as prescribed by the surgeon. All participants watched a tutorial video and practiced responding to a mock Panda alert.

Participants used Panda at home to manage their pain and track the regression of their PNB, including the presence or absence of complications such as paresthesias. Participants were asked to use Panda for a period between two and seven days. The identifying information, selection of pain scale, and details of the block were locked from changes by the participant; however, participants could add, change, or remove medications from the schedule. Alerts scheduled at inconvenient times, such as during 
Fig. 1 Panda-Nerve

Block home screen. PANDA =

Pain Assessment via a Novel

Digital App
Hello, Test No future medications scheduled. Your last pain check: 86/100

\section{No past medication}

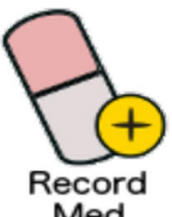

Upload

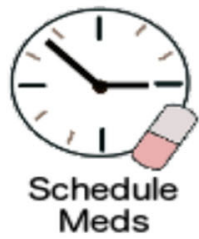

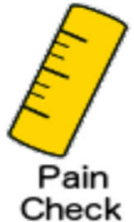

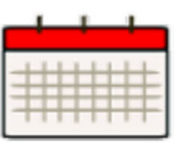

Calendar sleep, could be cancelled. All user data and activity within Panda were tracked and uploaded to REDCap.

Participants were contacted by phone or email $48 \mathrm{hr}$ after discharge as a check-in. Additionally, patients could contact the research assistant for help throughout the study. The anticipated last day of usage was determined by the patient on postoperative day 2 , but they could contact the research assistant if they finished using Panda sooner than expected. A standardized 14-question telephone interview was administered by the research assistant on the patient's last day of app use; a shorter five-question version was available for patients who did not have enough time for the full questionnaire and was used with one patient (Appendix 2). These questions were selected to identify major barriers to use and the perceived utility of the app for postoperative pain management. Participants additionally completed a standardized Computer Systems Usability Questionnaire (CSUQ) to assess the app's ease of use. ${ }^{18}$ Upon completion of the study, Panda was remotely deactivated and patients were instructed to delete the Panda app from their device.

Data analysis

After each round, telephone interviews and CSUQ responses were analyzed for common themes. Themes were used by the Panda software developer to direct modifications to Panda before the next round of the study. Qualitative analysis was performed using NVivo 12.2.0 (QSR International Pty Ltd., Melbourne, Victoria, Australia).

Each user's audit log was analyzed for the following values: the total study time, the median response time, the percentage of alerts to which a response was logged prior to the next alert in both the first $48 \mathrm{hr}$ of app usage and throughout the entire period of app usage, the proportion of logged responses that occurred within $60 \mathrm{~min}$, the median pain score (either VAS or NRS), the percentage of pain scores that were logged as no pain (either a response of "no pain" at the pain check prompt or a value of 0 on the scale), the number of times an analgesic medication was taken (defined as a non-steroidal anti-inflammatory drug, acetaminophen, or an opioid medication), and the median interval between analgesic medication administration. A one-hour response time was selected as it was felt to be the maximum allowable delay to still be considered compliant with the medication regime; this cut-off has been used by our group in the past. ${ }^{11,12}$ These data were collated for each individual round; the median [interquartile range (IQR)] of each parameter was calculated and the Kruskal-Wallis test was used to compare data between rounds. A $P$ value of less than 0.05 was considered significant. Calculations were performed using R Studio 1.1 (RStudio Inc., Boston, Massachusetts, USA).

\section{Results}

Cohort characteristics

Forty-one patients consented across three rounds (Fig. 2). Twenty-nine participants were analyzed in full.

Group characteristics can be seen in Table 1. Most patients reported having high or medium smartphone knowledge. The largest age group consisted of users over the age of 50 . No patients used an electronic method to keep track of medications already taken.

App usage

Across all three rounds, the median [IQR] proportion of alert responses before the next alert was 68 [34-93]\%. During the first $48 \mathrm{hr}$ of app use, the median [IQR] 
Fig. 2 Patient recruitment across all three rounds

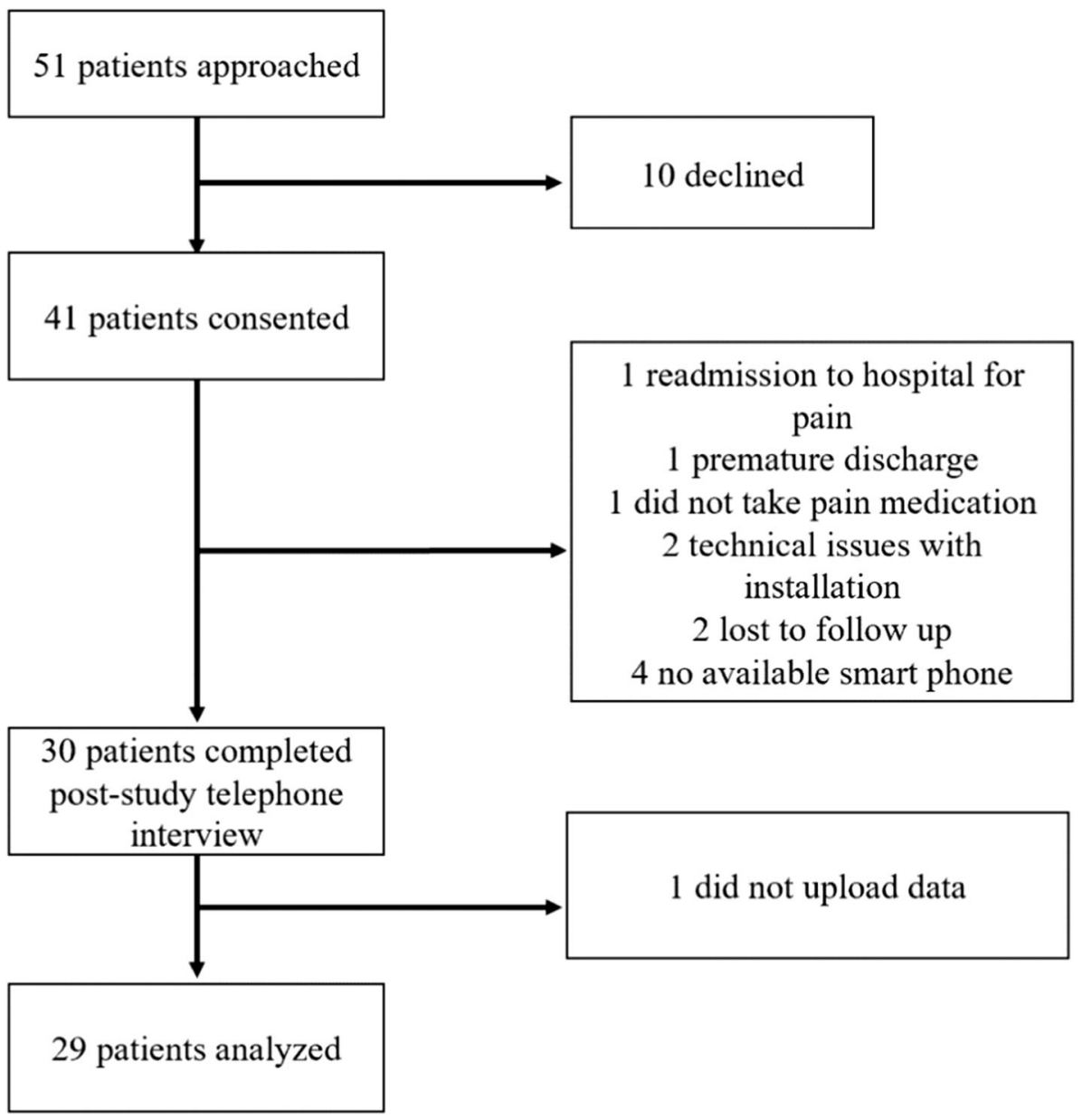

proportion of responses before the next alert was higher at 83 [54-92]\% (Table 3). Of the recorded responses, the median $[\mathrm{IQR}]$ proportion of responses within one hour was 87 [75-95]\%. There were no significant differences between groups for any usage parameter nor were differences found in median pain scores, pain scores recorded as no pain, doses of analgesic medication taken, or median interval between analgesic medications taken (Table 2). Only one patient required app assistance.

Twenty patients $(69 \%)$ completed the CSUQ. Across all three rounds, the median [IQR] response to "Overall, I am satisfied with this system" was 2 [1-3.5], equivalent to "agree". The most negative response was to the statement "This interface gives error messages that clearly tell me how to fix the problems" (4 [3-4.5]), equivalent to "neutral". The most positive response was to the statement "The interface is pleasant" (1.5 [1-2]). All other responses ranged between 2 and 3, corresponding to "agree".
User feedback and user-directed changes

Panda elicited positive responses from users (Table 4). Patients found the app easy to use and would use it again for a future surgery, with many specifically commenting that they liked the scheduling and reminder functions. Most patients felt they could set Panda up independently.

Responses to the layout and aesthetics of the app were mixed. Several requested changes to the app could not be made, including a self-adjusting, dynamic medication schedule that accounted for PRN doses and modifications to the pain scales, such as graduations to the VAS.

Several barriers to use were identified. Some patients required extensive assistance with installing the app on their device (e.g., resetting passwords, connecting to WiFi). One patient had difficulties using Panda and stopped after two alerts. A small number of technical glitches were also reported by patients, such as freezing or problems with saving entries.

These themes formed the basis of the changes made to Panda between rounds. One persistent concern was the language of the nerve block check. While patients could originally choose that their limb felt "normal", several 
TABLE 1 Study cohort characteristics

\begin{tabular}{|c|c|c|c|c|c|}
\hline \multicolumn{6}{|l|}{ Study cohort characteristics $(n=29)$} \\
\hline \multirow[t]{2}{*}{ Sex } & Male & Female & & & \\
\hline & $10(34 \%)$ & $19(66 \%)$ & & & \\
\hline \multirow[t]{2}{*}{ Age } & $<20$ & $20-29$ & $30-39$ & $40-49$ & $\geq 50$ \\
\hline & $2(7 \%)$ & $2(7 \%)$ & $5(17 \%)$ & $7(24 \%)$ & $13(45 \%)$ \\
\hline \multirow[t]{2}{*}{ Surgical site } & Upper extremity & Lower extremity & & & \\
\hline & $15(52 \%)$ & $14(48 \%)$ & & & \\
\hline \multirow[t]{2}{*}{ Operating system } & iOS & Android & & & \\
\hline & $21(72 \%)$ & $8(28 \%)$ & & & \\
\hline \multirow[t]{2}{*}{ Reported knowledge of smartphone use } & High & Medium & Low & None & \\
\hline & $12(41 \%)$ & $10(34 \%)$ & $6(21 \%)$ & $1(3 \%)$ & \\
\hline \multirow[t]{2}{*}{ Frequency of any app usage } & Daily & Weekly & Monthly & Never & \\
\hline & $17(59 \%)$ & $4(14 \%)$ & $5(17 \%)$ & $3(10 \%)$ & \\
\hline \multirow[t]{2}{*}{ Usage of health or fitness apps } & Yes & No & & & \\
\hline & $16(55 \%)$ & $13(45 \%)$ & & & \\
\hline \multirow[t]{2}{*}{$\begin{array}{l}\text { How do you remember to take your } \\
\text { medications? }\end{array}$} & Electronic reminder & Memory only & Physical reminder & $\begin{array}{c}\text { Never taken } \\
\text { medication } \\
\text { previously }\end{array}$ & \\
\hline & $11(38 \%)$ & $13(45 \%)$ & $2(7 \%)$ & $2(7 \%)$ & \\
\hline \multirow[t]{2}{*}{$\begin{array}{l}\text { How do you keep track of which } \\
\text { medications you have already taken? }\end{array}$} & Electronic reminder & Memory only & Physical reminder & $\begin{array}{c}\text { Never taken } \\
\text { medication } \\
\text { previously }\end{array}$ & \\
\hline & $0(0 \%)$ & $16(55 \%)$ & $11(38 \%)$ & $2(7 \%)$ & \\
\hline \multirow[t]{2}{*}{ Preferred method of pain reporting } & $\begin{array}{l}\text { Qualitative (e.g., "a } \\
\text { little"/"a lot" }\end{array}$ & $\begin{array}{l}\text { Quantitative } \\
\quad(\text { e.g., } 0-10)\end{array}$ & & & \\
\hline & $11(38 \%)$ & $18(62 \%)$ & & & \\
\hline
\end{tabular}

TABLE 2 Audit-trail log data analysis and calculated usage parameters per study round

\begin{tabular}{|c|c|c|c|c|}
\hline Usage parameter & Round 1 & Round 2 & Round 3 & $P$ \\
\hline Sample size $(n)$ & 9 & 10 & 10 & \\
\hline App usage time (hr) & $163.0[140.0-164.0]$ & $75.0[73.3-110.4]$ & $147.2[108.4-165.5]$ & 0.06 \\
\hline Percentage of alert responses & $52[28-78]$ & 89 [54-95] & $62[37-94]$ & 0.35 \\
\hline \multicolumn{5}{|l|}{ before next alert } \\
\hline Percentage of alert responses before next alert in first $48 \mathrm{hr}$ & $77[54-88]$ & 89 [73-93] & 75 [53-92] & 0.47 \\
\hline Response time (min) & $19[1-29.75]^{\mathrm{a}}$ & $1.5[0-6]$ & $0.5[0-3.25]$ & 0.42 \\
\hline Proportion of responses within $60 \mathrm{~min}(\%)$ & $79[60-88]^{\mathrm{a}}$ & $91[76-94]$ & 90 [78-99] & 0.32 \\
\hline Median pain score, VAS & $43[40-46]$ & $4[0-9.5]$ & $17[2-33]$ & 0.08 \\
\hline Median pain score, NRS & $3.5[3.0-3.5]$ & $5[4-7]$ & $2.5[2-4]$ & 0.40 \\
\hline Proportion of pain scores recorded as "no pain" (\%) & $17[5-26]^{\mathrm{a}}$ & 15 [3-83] & $21[8-41]$ & 0.78 \\
\hline Median recorded doses of analgesic taken $(n)$ & $14[4-31]$ & $9.5[6.5-14.8]$ & $30.5[11.5-33.0]$ & 0.37 \\
\hline Median interval between analgesic doses (min) & $242[240-336]^{\mathrm{a}}$ & 240 [228-293] & $240[240-272]$ & 0.48 \\
\hline
\end{tabular}

All values represent calculated medians; intervals represent interquartile range. Median response time and proportion of responses within 60 min does not include alerts to which no response was logged

a $n=8$, excludes one patient who did not respond to any alerts and for whom these parameters could not be calculated. NRS $=$ numerical rating scale; VAS = visual analogue scale 
TABLE 3 Compiled audit-trail log data analysis and calculated usage parameters across all three rounds

\begin{tabular}{lc}
\hline Usage parameter & 29 \\
\hline Sample size $(n)$ & $140.5[76.1-163.8]$ \\
App usage time $(\mathrm{hr})$ & $68[34-93]$ \\
Percentage of responses before next alert hours (\%) & $83[54-92]$ \\
Percentage of responses before next alert in the first $48 \mathrm{hr}(\%)$ & $1[0-13]^{\mathrm{b}}$ \\
Median response time (min) & $86.6[75.0-95.0]^{\mathrm{b}}$ \\
Proportion of responses within 60 min (\%) & $17[2-37]$ \\
Median pain score, VAS $(n=13)$ & $3.5[2.75-5.00]$ \\
Median pain score, NRS $(n=15)$ & $16[4-42]^{\mathrm{b}}$ \\
Proportion of pain scores recorded as “no pain" (\%) & $14[6-31]$ \\
Doses of analgesic taken & $240[237-316]^{\mathrm{b}}$ \\
Median interval between analgesic doses (min) &
\end{tabular}

All values represent calculated medians [interquartile range]

Median response time and proportion of responses within $60 \mathrm{~min}$ do not include alerts to which no response was logged

b $n=28$, excludes one patient who did not respond to any alerts and for whom these parameters could not be calculated

TABLE 4 Qualitative analysis results

\begin{tabular}{lc}
\hline Theme & Number of patients $(n=29)$ \\
\hline Found Panda-Nerve Block easy to use & 27 \\
Found it easy to respond to an alert & 26 \\
Liked the sound of the alarm & 25 \\
Would use Panda for a future surgery & 23 \\
Would feel comfortable setting up Panda without assistance & 23 \\
Liked having regular reminders and alerts & 14 \\
Logged an off-schedule medication & 11 \\
Edited a previous medication entry & 11 \\
Found the appearance childish or juvenile & 7 \\
Did not understand assessment of nerve block & 7 \\
Experienced difficulties navigating Panda & 5 \\
Requested a tablet counter within Panda & 4 \\
Used notepad function & 4 \\
Could not add custom medication & 4 \\
Requested a "smarter" app that updated the medication schedule dynamically & 3 \\
Found the app repetitive & 2 \\
Requested to use Panda in portrait orientation & 2
\end{tabular}

PANDA = Pain Assessment via a Novel Digital App

found this confusing as it did not feel normal compared with their pre-surgical baseline. The question was changed to ask if sensation in the operative limb was "returning" for the second round, and further changed to "returned" for the third round with good satisfaction.

Several changes were made to the set-up process. The option to add multiple PNBs was added at the end of the first round to account for combinations of blocks. The ability to enter a custom medication name was also added after the second round to better accommodate patients with multiple medications.

\section{Discussion}

Smartphone apps are appealing for ambulatory surgery as they guide patients through their postoperative recovery from home. Our study shows Panda is feasible and useable 
for patients to manage postoperative pain and monitor the regression of their nerve block following day surgery. We attribute this to the rounds of testing and iterative improvements conducted across multiple studies. ${ }^{11,12}$

Most patients found the app useful and responded appropriately to alerts. Additionally, response rates were high in the first $48 \mathrm{hr}$ post-discharge, capturing the critical transition from blockade to oral analgesia, including documentation of opioid usage and real-time nerve injury assessment.

Our app met our threshold response rate of 50\% and had improved adherence rates over the pediatric Panda app, showing Panda's feasibility. ${ }^{12}$ This may be because the user population was adult patients rather than parents or caregivers. Patients are inherently more aware of their symptoms and conceivably more motivated to use the app.

Several works on perioperative apps have been published. ${ }^{19-21}$ Warren-Stomburg et al. describe a postoperative pain app for ambulatory surgical patients. In contrast to our study, the implementation was complicated by low user adherence and high attrition. ${ }^{22}$ The authors hypothesized that this may have been due to a lack of feedback to the patient as well as user-side technical glitches.

Despite also not including a feedback system, usage of Panda was not affected by this. Conclusions about the differing adherence rates are challenging to draw because different metrics were used. The attrition rate may have been lower because Panda is a more comprehensive app that includes more features. Panda is also specifically developed for ambulatory PNB patients rather than all ambulatory patients, tailoring to specific needs of that population, again potentially improving buy-in. Additionally, Warren-Stomburg et al. did not perform follow-up, whereas we completed both a $48 \mathrm{hr}$ check-in and a final study interview.

\section{Barriers to usage}

As also observed in the pediatric study, ${ }^{12}$ some patients requested a version of the app that would automatically shift the future medication schedule when they took their medication late. This would require a smart algorithm to prevent complications such as unsafe dosing intervals and medication overdose, which was beyond the scope of this initial usability/feasibility study. Requests to modify the pain scales, such as adding graduations to the VAS, could also not be accommodated as deviation from these scales would require re-validation.

Iterative improvements to the app did not significantly change its usability. The most common user critique, the phrasing of the nerve block check, was resolved by the last round. Most patients were able to install, set-up, and use the app; nevertheless, there were several patients who required extensive assistance in the initial set-up. While feasible in the context of a study, this degree of assistance is impractical for a busy clinician. With respect to the two patients who could not be enrolled because of technical issues, we were unable to reproduce these glitches, which may have been device-specific.

The lack of significant differences in usage metrics between rounds suggests that the initial version, though not completely optimized, was itself useable for patients, probably because many of the challenges in implementation were addressed in the original pediatric Panda study. User criticism was generally centred around aesthetic concerns rather than functionality, but this may have impacted user adherence and motivation. While our 
study is underpowered to detect subtle differences, statistically significant changes were seen between rounds in the similarly sized pediatric study. ${ }^{12}$

\section{Limitations of data}

A limitation of this study is the reliance on self-reported data. Attrition bias is also a concern: patients may have been lost to follow-up because they found Panda uninteresting or unhelpful. While not analyzed in full, multiple days of data were still submitted for these patients, suggesting they continued to use the app. The Hawthorne effect must be considered; outside of a study, patient adherence could diminish in clinical practice.

Although the VAS and NRS are well-established, and we have shown agreement between the paper and electronic versions of these scales in the recovery room, the electronic versions of these scales have not specifically been validated for at-home patients. ${ }^{13-15}$

Future directions

Our work is a step toward providing improved pain management at home for ambulatory surgical patients via apps. As we move toward implementing the app into routine postoperative care, we anticipate publishing Panda to the public iOS and Android app stores, simplifying installation. Moreover, we are developing additional features including a two-way communication system between clinicians and patients. Patients will be able to communicate directly to a clinician either through text, voice, or video messaging, including photographs of their surgical sites and peripheral nerve catheters.
In addition to its primary goal of improving pain management for ambulatory patients, Panda has potential for assisting in diagnosing PNB-related complications. Real-time postoperative follow-up does not just provide detailed records for clinicians but facilitates early diagnosis of complications. Through regular screening for concerning symptoms following a PNB, Panda can assist the early diagnosis of nerve injuries, compressive hematomas, and abscesses, on which more timely interventions can be delivered.

While effects on clinical patient outcomes were beyond the scope of our study, Panda could potentially play a role in reducing periods of severe pain and decreasing readmissions to hospital; we anticipate testing these hypotheses in a future randomized-controlled trial.

Author contributions Alexander Dotto contributed to the data acquisition, data analysis, and manuscript writing. Dustin Dunsmuir contributed to the conception of the study, development of the app, interpretation of data, and manuscript writing. Terri Sun contributed to the conception of the study, protocol design, data analysis and interpretation, and manuscript writing. Lily Chiu contributed to the protocol design and manuscript writing. Ron Ree, J. Mark Ansermino, and Cynthia Yarnold contributed to the conception of the study, data interpretation, and editing of the manuscript.

Acknowledgements The authors would like to thank the staff of St. Paul's Hospital for their assistance.

Conflicts of interest None.

Funding statement This study was funded in part by Thrive Health who provided an unrestricted grant. Thrive Health did not have any input on the content of this manuscript.

Editorial responsibility This submission was handled by Dr. Philip M. Jones, Associate Editor, Canadian Journal of Anesthesia. 
APPENDIX 1 Pre-study questionnaire

Panda at home feasibility-pre-study questionnaire

Thank you for participating. Please circle answers to questions 1-10

and give brief written answers to questions 11-13.

1. Age (years): $\quad<20 \quad 20-30 \quad 30-40 \quad 40-50 \quad>50$

2. Sex: $M \quad F$

3. Do you own and operate a smartphone?

Yes No If yes, what mobile device do you own?

4. How do you consider your mobile phone knowledge level?
High
Medium
Low
None

5. How often do you use your mobile phone to make, receive phone calls?
Daily
A few times a week
A few times a month
Never

6. How often do you use your mobile phone for text messaging?
Daily
A few times a week
A few times a month
Never

7. How often do you use your mobile phone for emails and surfing?
Daily
A few times a week
A few times a month
Never

8. How often do you use your mobile phone apps?
Daily
A few times a week
A few times a month
Never

9. What sorts of apps do you normally use on your smartphone? Check all that apply:
Games/entertainment
Health/fitness
Education/news
Social media
I don't use apps

To what level do you agree or disagree with the following statement?

10. I can see advantages for in using mobile apps to help manage postoperative pain
Strongly agree
Agree
Disagree
Strongly disagree

11. How you would normally remind yourself to take medications (e.g., by memory, set alarm, etc.)?

12. How you would normally keep track of the medications you have taken (i.e., by memory, written note, electronic note, excel spreadsheet, etc.)?

13. How you would normally assess your pain (i.e., scale of $0-10$, a little/a lot, etc.)? 


\section{APPENDIX 2 Post-study telephone questionnaire}

The purpose of this interview is to obtain as much feedback as possible from the participant. This should take no more than $10-15$ min

\section{Preamble}

a. Confirm you are speaking to the right person, i.e., consented and main user of the Panda app during the study period; otherwise, re-arrange.

b. Thank them for their participation in the study.

c. Confirm that their study data has been uploaded successfully and received by the research team.

d. Explain the format of the post-study interview.

e. Confirm they are willing to talk with you for 10-15 min and that now is a good time; otherwise re-arrange.

f. Explain that you will be transcribing the interview verbatim (with the identifying information omitted), and confirm that this is OK.

g. Record date and time started/finished.

\section{Short interview questions (general, if time limited)}

1. How easy was it to use this app?

2. Do you see yourself using this app? Why or why not?

3. What did you like least about the app? What did you like most?

4. Do you think the app can do more? If so, what additional functions would you like to see?

5. Any other feedback?

Extended interview questions (detailed, if time permits)

Directed questions based on user log function

1. Generally, what did you think about using the app? (Easy or difficult?)

2. What did you like the least or find most difficult about using the app?

3. What did you like the most about using the app?

4. What would you add or change to improve the app? Do you think the app could do more?

a. What do you think about the appearance or layout of the app and how could this be improved?

5. What did you think about setting up the app? How would you feel doing this on your own at home?

6. What did you think about the "do not disturb" function?

7. In terms of the medication alert pop-up which said: "Skip", "Snooze" and "Proceed"

a. What option do you think you chose most often?

b. Did you feel you knew how to respond to a medication alert? Was this clear? c. What do you think about the alert sound?

d. If you used the "Snooze" function, did you use the "Respond Now" button? Could you find the "Respond Now" button or were you aware of what this button was for?

8. What did you think about the pain check function?

e. When did you do pain-checks? (Giving medications or off-schedule)

f. How easy was it to do?

g. How useful was this function?

9. When confirming a medication is taken the app asks "have you given additional medication in the last ' $\mathrm{X}$ ' hours?" Yes or No? What do you think about this question? Was it easy or difficult to understand?

10. What did you think about editing medications or redoing pain scores from the calendar page?

h. Did you use this function, could you find it, and how easy was it to use?

i. How useful was this function?

11. What did you think about adding notes to a pain score or medication from the calendar page?

j. Did you use this function, could you find it, and how easy was it to use?

k. How useful was this function?

12. What did you think about recording off-schedule medications?

1. Did you use this function, could you find it, and how easy was it to use?

m. How useful was this function?

13. Overall, what do you think about using this app? What would motivate you to use the app?

14. Do you have any other feedback?

\section{References}

1. Liu SS, Strodtbeck WM, Richman JM, Wu CL. A comparison of regional versus general anesthesia for ambulatory anesthesia: a meta-analysis of randomized controlled trials. Anesth Analg 2005; 101: 1634-42.

2. Sunderland S, Yarnold CH, Head SJ, et al. Regional versus general anesthesia and the incidence of unplanned health care resource utilization for postoperative pain after wrist fracture surgery: results from a retrospective quality improvement project. Reg Anesth Pain Med 2016; 41: 22-7.

3. McCartney CL, Brull R, Chan VW, et al. Early but no long-term benefit of regional compared with general anesthesia for ambulatory hand surgery. Anesthesiology 2004; 101: 461-7.

4. Abdallah FW, Halpern SH, Aoyama $K$, Brull $R$. Will the real benefits of single-shot interscalene block please stand up? A 
systematic review and meta-analysis. Anesth Analg 2015; 120: 1114-29.

5. Dayer L, Heldenbrand S, Anderson P, Gubbins PO, Martin BC. Smartphone medication adherence apps: potential benefits to patients and providers. J Am Pharm Assoc 2013; 53: 172-81.

6. Cumino O, Vieira JE, Lima LC, Stievano LP, Silva RA, Mathias $L A$. Smartphone-based behavioural intervention alleviates children's anxiety during anaesthesia induction: a randomised controlled trial. Eur J Anaesthesiol 2017; DOI: https://doi.org/10. 1097/EJA.0000000000000589.

7. Davis SR, Peters D, Calvo RA, Sawyer SM, Foster JM, Smith L. “ Kiss myAsthma ": using a participatory design approach to develop a self-management app with young people with asthma. J Asthma 2018; 55 1018-27.

8. Kirwan M, Vandelanotte C, Fenning A, Duncan MJ. Diabetes Self-management smartphone application for adults with type 1 diabetes : randomized controlled trial. J Med Internet Res 2013; DOI: https://doi.org/10.2196/jmir.2588.

9. Pecorelli N, Fiore JF Jr, Kaneva P, et al. An app for patient education and self-audit within an enhanced recovery program for bowel surgery : a pilot study assessing validity and usability. Surg Endosc 2018; 32: 2263-73.

10. Brown NJ, Kimble RM, Rodger S, Ware RS, Cuttle L. Play and heal : randomized controlled trial of Ditto ${ }^{\mathrm{TM}}$ intervention efficacy on improving re-epithelialization in pediatric burns. Burns 2014; 40: 204-13.

11. Sun T, Dunsmuir D, Miao I, et al. In-hospital usability and feasibility evaluation of Panda, an app for the management of pain in children at home. Paediatr Anaesth 2018; 28: 897-905.

12. Dunsmuir $D, W u H$, Sun $T$, et al. A postoperative pain management mobile app (Panda) for children at home after discharge: usability and feasibility. JMIR Perioper Med 2019; DOI: https://doi.org/10.2196/12305.

13. Katz J, Melzack R. Measurement of pain. Surg Clin North Am 1999; 79: 231-52.

14. Hjermstad MJ, Fayers PM, Haugen DF, et al. Studies comparing numerical rating scales, verbal rating scales, and visual analogue scales for assessment of pain intensity in adults: a systematic literature review. J Pain Symptom Manage 2011; 41: 1073-93.

15. Chiu $L Y$, Sun T, Ree R, et al. The evaluation of smartphone versions of the visual analogue scale and numeric rating scale as postoperative pain assessment tools: a prospective randomized trial. Can J Anesth 2019; DOI: https://doi.org/10.1007/s12630019-01324-9.

16. Stinson JN, Jibb LA, Nguyen C, et al. Development and testing of a multidimensional iphone pain assessment application for adolescents with cancer. J Med Internet Res 2013; DOI: https:// doi.org/10.2196/jmir.2350.

17. Harris PA, Taylor R, Thielke R, Payne J, Gonzalez N, Conde JG. Research electronic data capture (REDCap) — A metadata-driven methodology and workflow process for providing translational research informatics support. J Biomed Inform 2008; 42: 377-81.

18. Lewis JR. IBM Computer usability satisfaction questionnaires: psychometric evaluation and instructions for use. Int $\mathrm{J}$ Hum Comput Interact 1995; 1: 57-78.

19. Gough AT, Fieraru G, Gaffney PA, Butler M, Kincaid RJ, Middleton $R G$. A novel use of $\mathrm{QR}$ code stickers after orthopaedic cast application. Ann R Coll Surg Engl 2017; 99: 476-8.

20. Wu J, Yu H, Ho T, Su X, Lin M, Lai F. Tablet PC-enabled application intervention for patients with gastric cancer undergoing gastrectomy. Comput Methods Programs Biomed 2015; 119: 101-9.

21. Gunter RL, Fernandes-Taylor S, Rahman S, et al. Feasibility of an image-based mobile health protocol for postoperative wound monitoring. J Am Coll Surg 2017; 226: 277-86.

22. Warren-Stomburg M, Jacobsson J, Brattwall M, Jildenstål P. Athome monitoring after surgery/anaesthesia - a challenge. J Eval Clin Pract 2016; 22: 882-6.

Publisher's Note Springer Nature remains neutral with regard to jurisdictional claims in published maps and institutional affiliations. 